\title{
Sequential changes in Rotterdam CT scores related to outcomes for patients with traumatic brain injury who undergo decompressive craniectomy
}

\author{
Kenji Fujimoto, MD, ${ }^{1,3}$ Masaki Miura, MD, PhD, ${ }^{1}$ Tadahiro Otsuka, MD, $\mathrm{PhD},{ }^{2}$ and \\ Jun-ichi Kuratsu, MD, PhD ${ }^{3}$
}

\begin{abstract}
'Department of Neurosurgery, Japanese Red Cross Kumamoto Hospital, Higashiku; '2Department of Neurosurgery, National Hospital Organization Kumamoto Medical Center, Chuo-ku; and '3Department of Neurosurgery, Faculty of Life Sciences, Kumamoto University School of Medicine, Chuo-ku, Kumamoto, Japan
\end{abstract}

\begin{abstract}
OBJECTIVE Rotterdam CT scoring is a CT classification system for grouping patients with traumatic brain injury (TBI) based on multiple CT characteristics. This retrospective study aimed to determine the relationship between initial or preoperative Rotterdam CT scores and TBI prognosis after decompressive craniectomy (DC).

METHODS The authors retrospectively reviewed the medical records of all consecutive patients who underwent DC for nonpenetrating TBI in 2 hospitals from January 2006 through December 2013. Univariate and multivariate logistic regression and receiver operating characteristic (ROC) curve analyses were used to determine the relationship between initial or preoperative Rotterdam CT scores and mortality at 30 days or Glasgow Outcome Scale (GOS) scores at least 3 months after the time of injury. Unfavorable outcomes were GOS Scores 1-3 and favorable outcomes were GOS Scores 4 and 5 .
\end{abstract}

RESULTS A total of 48 cases involving patients who underwent DC for TBI were included in this study. Univariate analyses showed that initial Rotterdam CT scores were significantly associated with mortality and both initial and preoperative Rotterdam CT scores were significantly associated with unfavorable outcomes. Multivariable logistic regression analysis adjusted for established predictors of TBI outcomes showed that initial Rotterdam CT scores were significantly associated with mortality (OR 4.98, 95\% Cl 1.40-17.78, $\mathrm{p}=0.01)$ and unfavorable outcomes (OR 3.66, 95\% Cl 1.29-10.39, $p=0.02$ ) and preoperative Rotterdam CT scores were significantly associated with unfavorable outcomes (OR 15.29, $95 \% \mathrm{Cl} 2.50-93.53, p=0.003$ ). ROC curve analyses showed cutoff values for the initial Rotterdam CT score of 5.5 (area under the curve [AUC] $0.74,95 \% \mathrm{Cl} 0.59-0.90, p=0.009$, sensitivity $50.0 \%$, and specificity $88.2 \%$ ) for mortality and 4.5 (AUC $0.71,95 \% \mathrm{Cl} 0.56-0.86, p=0.02$, sensitivity $62.5 \%$, and specificity $75.0 \%$ ) for an unfavorable outcome and a cutoff value for the preoperative Rotterdam CT score of 4.5 (AUC $0.81,95 \% \mathrm{Cl} 0.69-0.94, \mathrm{p}<0.001$, sensitivity $90.6 \%$, and specificity $56.2 \%$ ) for an unfavorable outcome.

CONCLUSIONS Assessment of changes in Rotterdam CT scores over time may serve as a prognostic indicator in TBI and can help determine which patients require DC.

http://thejns.org/doi/abs/10.3171/2015.4.JNS142760

KEY WORDS decompressive craniectomy; Glasgow Outcome Scale; mortality; Rotterdam CT score; traumatic brain injury

$\mathrm{T}$ RAUMATIC brain injury (TBI) is a major cause of death and neurological disability worldwide and thus is an important societal problem. Decompressive craniectomy (DC) is commonly performed for TBI patients with diffuse brain swelling or a refractory increase in intracranial pressure (ICP). The beneficial effects of DC for reducing ICP have been well documented in several stud- ies, ${ }^{1,2,24}$ and DC can also contribute to improvements in cerebral compliance, oxygenation, and blood flow. . $^{12,14,25,28}$ Currently, however, there is no evidence based on prospective controlled randomized trials to establish that DC actually improves these patients' outcomes. ${ }^{5}$ Thus, any beneficial effects of DC remain controversial. With regard to clinical decision making, counseling patients' families,

ABBREVIATIONS AUC = area under the curve; $\mathrm{DC}=$ decompressive craniectomy; $\mathrm{GCS}=$ Glasgow Coma Scale; GOS = Glasgow Outcome Scale; ICP = intracranial pressure; ROC = receiver operating characteristic; $\mathrm{TBI}=$ traumatic brain injury.

SUBMITTED December 5, 2014. ACCEPTED April 8, 2015.

INCLUDE WHEN CITING Published online October 23, 2015; DOI: 10.3171/2015.4.JNS142760. 
and resource allocation, there is a need for reliable methods of predicting outcome before performing DC for TBI.

Rotterdam CT scoring is a CT classification system that was developed by Maas et al. in 2005 to group patients with TBI based on multiple CT characteristics (Table 1). ${ }^{18}$ These scores combine individual CT characteristics, including any midline shift, the status of the basal cisterns, and the types of mass lesions or intracranial hemorrhage. Rotterdam CT scores are easily derived, and the interobserver reproducibility is very good between multiple readers with varied backgrounds. ${ }^{3}$ A number of reports have shown that these scores can predict the outcome of TBI. $7,9,13,15,17,19,22,23$ However, this has not been clearly established for TBI patients who undergo DC. In addition, no studies have assessed the optimal timing for determining Rotterdam CT scores prior to DC as a prognostic predictor.

Therefore, in the present study, we retrospectively collected clinical data and determined the relationship between initial or preoperative Rotterdam CT scores and prognosis of TBI in patients who underwent DC.

\section{Methods}

\section{Patients and Clinical Data}

We retrospectively reviewed the medical records of all consecutive patients who underwent a unilateral decompressive hemicraniectomy for nonpenetrating TBI in 2 hospitals with emergency medical care centers, Japanese Red Cross Kumamoto Hospital and National Hospital Organization Kumamoto Medical Center, in Japan from January 2006 through December 2013. We excluded patients who had bilateral hemicraniectomy, bifrontal hemicraniectomy, penetrating TBI, or a prior surgical procedure to evacuate a space-occupying lesion and subsequently had delayed brain swelling.

Patient data collected included age, sex, preoperative Glasgow Coma Scale (GCS) score, preoperative pupil reactivity, lesion location, time from head injury to DC, head Abbreviated Injury Scale (AIS) score, Injury Severity Score (ISS), international normalized ratio of prothrombin time (PT-INR), use of anticoagulants and/or antiplatelet agents prior to head injury, previous medical history, mechanism of trauma incurring injury, and initial and preoperative Rotterdam CT scores.

\section{CT Scanning and Rotterdam CT Scores}

CT scanning was performed as soon as possible after a patient arrived in the emergency department. Follow-up CT scans were performed in cases in which focal neurological deficits appeared, for patients with progressively disturbed consciousness, or for those with no neurological improvement. Initial and preoperative CT scans were scored using the Rotterdam CT scoring system as follows (Table 1): a) basal cistern status was classified as normal (0), compressed (1), or absent (2); b) midline shift was classified as 0-5 mm (0) or > $5 \mathrm{~mm}(1)$; c) epidural hematoma was classified as present (0) or absent (1); and d) traumatic subarachnoid hemorrhage or/and intraventricular hemorrhage was classified as absent (0) or present (1). One point was added to the summed score to make this grading numerically consistent with the Marshall CT classification, ${ }^{21}$ which classifies TBI into 6 categories.
TABLE 1. Rotterdam CT scoring system*

\begin{tabular}{cc}
\hline \multicolumn{1}{c}{ CT Finding } & Score \\
\hline Basal cisterns & \\
\hline Normal & 0 \\
\hline Compressed & 1 \\
\hline Absent & 2 \\
\hline Midline shift & 0 \\
\hline$\leq 5 \mathrm{~mm}$ & 1 \\
\hline$>5 \mathrm{~mm}$ & \\
\hline Epidural mass lesion & 0 \\
\hline Present & 1 \\
\hline Absent & 0 \\
\hline Intraventricular or subarachnoid hemorrhage & 1 \\
\hline Absent & +1 \\
\hline Present &
\end{tabular}

* Based on the study by Maas et al. ${ }^{18}$

\section{Decompressive Craniectomy}

Decompressive craniectomy was performed using standardized procedures. The anteroposterior diameter of a bone flap was approximately $12-15 \mathrm{~cm}$, with extension into the temporal base. The dura mater was opened, and this opening was extended to the bone margins. The brain surface was loosely covered by the remaining dura or an artificial dural substitute without a closure.

\section{Outcome Measures}

Our outcome measures were mortality at 30 days and Glasgow Outcome Scale (GOS) scores recorded at least 3 months after the time of injury. The neurological outcomes in cases with long-term follow-up were assessed at the time when neurological findings had adequately improved. Unfavorable outcomes were defined as GOS Scores 1-3 and favorable outcomes were GOS Scores 4 and 5.

\section{Statistical Analysis}

Statistical analyses were performed using SPSS software (version 19, IBM Corp.). Results are presented as means \pm standard deviations. Possible relationships between Rotterdam CT scores and prognosis after DC for TBI were first analyzed by binary logistic regression analysis, after which multivariable logistic regression models were generated to adjust for established predictors of outcomes after TBI (e.g., age, GCS score, and pupil reactivity). ${ }^{1,6,9,10,15,16,20}$ A $\mathrm{p}$ value $<0.05$ by multivariate analysis was considered significant. To identify the optimal cutoff values for the continuous variables of significant factors in multivariate analysis, we performed receiver operating characteristic (ROC) curve analyses.

\section{Results \\ Patient Characteristics}

From January 2006 through December 2013, a total of 2022 patients who had moderate or severe TBI were admitted, of whom 49 had undergone unilateral decompressive hemicraniectomy for TBI and met our inclusion 
criteria. Of these 49 patients, one had been injured immediately after a cerebellar infarction, which was critical and resulted in death. Therefore, we excluded this patient and finally assessed the remaining 48 patients. These patients' characteristics are summarized in Table 2. Our series included 28 men and 20 women, with a mean age of $54.5 \pm$ 22.0 years.

\section{Rotterdam CT Scores}

Follow-up CT scans were performed for 22 patients, while 26 patients had undergone DC immediately after an initial CT scan. Therefore, for these 26 patients, their initial Rotterdam CT scores were equal to their preoperative Rotterdam CT scores. Preoperative Rotterdam CT scores were the worst for 47 patients, whereas for 1 patient, the initial Rotterdam CT score was lower than the preoperative Rotterdam CT score because of spontaneous resolution of a subdural hematoma.

All patients underwent DC on the basis of a comprehensive assessment of neurological findings (e.g., consciousness and pupillary reaction) and CT findings (e.g., midline shift and size of brain contusion); no patient had an ICP monitoring device placed for preoperative monitoring.

\section{Patient Outcomes}

Fourteen (29\%) of the 48 patients who underwent DC died within the first 30 days. Seven of these patients died during the first 7 days and the other 7 died within 7-30 days after DC. Thirty-four patients (71\%) survived and were followed up for at least 3 months after treatment. Ten patients (21\%) showed a good recovery (GOS Score 5) and social reintegration, and $6(13 \%)$ had moderate deficits (GOS Score 4). Six patients (13\%) exhibited severe deficits (GOS Score 3), and $12(25 \%)$ remained in a vegetative state (GOS Score 2). Overall, 16 patients (33\%) had a favorable outcome and $32(67 \%)$ had an unfavorable outcome.

\section{Rotterdam CT Scores and Patient Prognosis}

Table 3 shows the relationship between Rotterdam CT scores and prognosis after DC in our patient group. Patients with higher Rotterdam CT scores tended to have a poorer outcome based on both initial and preoperative CT scans. The mortality rate of patients with a preoperative Rotterdam CT score of 6 (44\% of the patients) was lower than that of the patients with an initial score of $6(64 \%)$, because none of the patients who died had a Rotterdam CT score of 6 after the preoperative CT scans. In addition, the mortality rate of those patients who underwent preoperative CT scans was $9 \%(2 / 22)$.

Results of univariate analyses showed that initial Rotterdam CT scores were significantly associated with mortality $(\mathrm{p}=0.01)$ and both initial $(\mathrm{p}=0.02)$ and preoperative $(\mathrm{p}=0.002)$ Rotterdam CT scores were significantly associated with an unfavorable outcome. Multivariable logistic regression analysis adjusted for established predictors of TBI outcomes, including age, GCS score, and pupil reactivity, showed that initial Rotterdam CT scores were significantly associated with both mortality (OR 4.98, 95\% CI 1.40-17.78, $\mathrm{p}=0.01)$ and an unfavorable outcome (OR $3.66,95 \%$ CI $1.29-10.39, \mathrm{p}=0.02$ ), and preoperative Rotterdam CT scores were significantly associated with an
TABLE 2. Summary of demographic and clinical characteristics of 48 patients*

\begin{tabular}{|c|c|}
\hline Variable & Value \\
\hline Age in yrs (mean \pm SD) & $54.5 \pm 22.0$ \\
\hline Male sex & $28(58)$ \\
\hline Initial GCS score $\leq 6$ & $18(38)$ \\
\hline Preop GCS score $\leq 6$ & $33(69)$ \\
\hline Initial abnormal pupil reaction & $23(48)$ \\
\hline Preop abnormal pupil reaction & $41(85)$ \\
\hline Left-sided lesion & $18(38)$ \\
\hline Timing of DC (<24 hrs) & $44(92)$ \\
\hline Head AIS score (mean \pm SD) & $4.7 \pm 0.5$ \\
\hline ISS (mean \pm SD) & $23.8 \pm 7.6$ \\
\hline PT-INR (mean \pm SD) & $1.1 \pm 0.2$ \\
\hline Antiplatelet agent use & $2(4)$ \\
\hline Warfarin use & $1(2)$ \\
\hline \multicolumn{2}{|l|}{ Medical history } \\
\hline Cerebrovascular disease & $2(4)$ \\
\hline Ischemic cardiac disease & $0(0)$ \\
\hline Deterioration in liver function & $3(6)$ \\
\hline Terminal renal failure requiring dialysis & $0(0)$ \\
\hline Diabetes mellitus & $6(13)$ \\
\hline Hypertension & $12(25)$ \\
\hline \multicolumn{2}{|l|}{ Mechanism of injury } \\
\hline Traffic accident & $17(35)$ \\
\hline Fall & $29(60)$ \\
\hline Other & $2(4)$ \\
\hline
\end{tabular}

AIS = Abbreviated Injury Scale; ISS = Injury Severity Score; PT-INR = international normalized ratio of prothrombin time.

* Values represent numbers of patients (\%) unless otherwise indicated.

unfavorable outcome (OR 15.29, 95\% CI 2.50-93.53, $\mathrm{p}=$ 0.003 ) (Tables 4 and 5). Moreover, the multivariable logistic regression analyses identified that advanced age was significantly associated with both mortality (OR 1.07, 95\% CI $1.01-1.13, \mathrm{p}=0.02$ in initial score analysis; OR 1.05 , 95\% CI 1.01-1.10, $\mathrm{p}=0.03$ in preoperative score analysis) and an unfavorable outcome (OR 1.07, 95\% CI 1.02-1.12, $\mathrm{p}=0.01$ in initial score analysis; OR $1.10,95 \%$ CI $1.03-$ $1.18, p=0.007$ in preoperative score analysis), and initial GCS score $\leq 6$ was significantly associated with mortality (OR 18.58, 95\% CI 1.17-294.3, $\mathrm{p}=0.04$ ).

Because Rotterdam CT score and age were significantly associated with mortality and an unfavorable outcome, ROC curve analyses were conducted to determine the cutoff values. Results showed that the cutoff values for initial Rotterdam CT score were 5.5 (area under the curve [AUC] $0.74,95 \%$ CI $0.59-0.90, p=0.009$, sensitivity $50.0 \%$, and specificity $88.2 \%$ ) for mortality and 4.5 (AUC $0.71,95 \%$ CI $0.56-0.86, p=0.02$, sensitivity $62.5 \%$, and specificity $75.0 \%$ ) for an unfavorable outcome, and that of preoperative Rotterdam CT score was 4.5 (AUC 0.81, 95\% CI $0.69-0.94, p<0.001$, sensitivity $90.6 \%$, and specificity $56.2 \%$ ) for an unfavorable outcome. The cutoff age values were 62.5 years (AUC 0.77, 95\% CI 0.62-0.92, p $=0.004$, sensitivity $78.6 \%$, and specificity $70.6 \%$ ) for mortality and 
TABLE 3. Relationship between Rotterdam CT scores and mortality and unfavorable outcomes*

\begin{tabular}{cccc}
\hline $\begin{array}{c}\text { Rotterdam } \\
\text { CT Score }\end{array}$ & All Pts & $\begin{array}{c}\text { Pts Who Died w/in } \\
\text { 30 Days }\end{array}$ & $\begin{array}{c}\text { Pts w/ Unfavorable } \\
\text { Outcome }\end{array}$ \\
\hline Initial & & & \\
\hline 1 & $1(2)$ & $0(0)$ & $0(0)$ \\
\hline 2 & $4(8)$ & $0(0)$ & $1(25)$ \\
\hline 3 & $7(15)$ & $1(14)$ & $6(86)$ \\
\hline 4 & $12(25)$ & $3(25)$ & $5(42)$ \\
\hline 5 & $13(27)$ & $3(23)$ & $10(77)$ \\
\hline 6 & $11(23)$ & $7(64)$ & $10(91)$ \\
\hline Preop & & & $0(0)$ \\
\hline 1 & $0(0)$ & $0(0)$ & $0(0)$ \\
\hline 2 & $2(4)$ & $0(0)$ & $0(0)$ \\
\hline 3 & $0(0)$ & $0(0)$ & $3(30)$ \\
\hline 4 & $10(21)$ & $2(20)$ & $14(70)$ \\
\hline 5 & $20(42)$ & $5(25)$ & $15(94)$ \\
\hline 6 & $16(33)$ & $7(44)$ &
\end{tabular}

Pts = patients.

* Values represent numbers of patients (\%).

50 years (AUC 0.80, 95\% CI 0.66-0.94, $\mathrm{p}=0.001$, sensitivity $84.4 \%$, and specificity $68.7 \%$ ) for an unfavorable outcome.

\section{Discussion}

Our results indicated that Rotterdam CT scores were effective predictors for TBI patients who required DC. The severity of initial CT findings as assessed by Rotterdam CT scores was associated with both early death and an unfavorable outcome at least 3 months after the time of injury. In particular, initial Rotterdam scores of 6 were associated with early death, and the scores of 5 or 6 were associated with an unfavorable outcome. In comparison, preoperative Rotterdam CT scores were associated with an unfavorable outcome, particularly scores of 5 or 6 , but not with early death. The higher odds ratio for preoperative Rotterdam CT scores and unfavorable outcomes determined by multivariable logistic regression analysis indicated that the preoperative Rotterdam CT score was more effective than the initial Rotterdam CT score for predicting an unfavorable outcome. In addition, our results showed that age $\geq 63$ years and initial GCS score $\leq 6$ were associated with early death, and age $>50$ years was associated with an unfavorable outcome. Thus, the TBI pa- tients with Rotterdam CT scores $\leq 4$, under the age of 50 years, and with initial GCS scores $\geq 7$ are more likely to have a favorable outcome after DC.

The clinical severity of TBI is generally classified as severe, moderate, or mild based on the level of consciousness as assessed by the GCS. GCS scores have been shown to be significantly correlated with the TBI outcomes in patients who are treated with DC and in those who are not. $1,6,8,10,15,16,20$ However, determining the GCS score on admission is often difficult because patient with severe TBI are frequently sedated early on, intubated, and then treated with mechanical ventilation. Moreover, there are several factors that can cause consciousness deterioration in TBI patients, such as drinking alcohol, having a primary disease, or being in poor general condition..$^{27,29}$

CT scanning plays an important role in rapidly assessing acute-phase TBI because it can reveal posttraumatic hemorrhagic lesions and provide essential diagnostic information for surgical intervention. In 1991, Marshall et al. ${ }^{21}$ proposed a CT classification to group TBI patients based on CT characteristics. Several studies have confirmed the predictive value of this CT classification, ${ }^{11,13,22,23,26}$ and international guidelines on prognosis include this CT classification as a major CT predictor. However, in the CT classification of Marshall et al., almost all the patients who undergo DC are classified into only 2 categories, Class IV or Class V, because a decompressive procedure is accompanied by evacuating a mass lesion in nearly all cases. ${ }^{4}$ This means that the discriminatory capability of the CT classification of Marshall et al. is limited for TBI patients who require DC.

In 2005, Maas et al. ${ }^{18}$ developed an alternative CT classification, known as the Rotterdam CT scoring system, by determining the relationship between admission CT examination results and mortality at 6 months postinjury. They showed better prognostic discrimination for Rotterdam CT scores than for the CT scoring system of Marshall et al., particularly for patients with mass lesions.

Several studies that included not only surgical interventions but also conservative therapy revealed that Rotterdam CT scores were used as prognostic predictors for TBI patients. ${ }^{13,15,19,22,23}$ However, only a few studies have assessed the performance of Rotterdam CT scores for TBI patients who underwent DC. Huang et al..$^{9}$ indicated that the worst Rotterdam CT score prior to DC was an independent predictor of an unfavorable outcome and not of mortality at the end of follow-up as determined by multivariate logistic regression analysis. In addition, Flint et al. ${ }^{7}$ found that the severity of an initial Rotterdam CT score was associated with the risk of contusion expansion after

TABLE 4. Multivariate logistic regression analysis results for initial Rotterdam CT score associations with mortality and an unfavorable outcome

\begin{tabular}{|c|c|c|c|c|c|c|}
\hline \multirow[b]{2}{*}{ Variable } & \multicolumn{3}{|c|}{ Mortality } & \multicolumn{3}{|c|}{ Unfavorable Outcome } \\
\hline & OR & $95 \% \mathrm{Cl}$ & $p$ Value & OR & $95 \% \mathrm{Cl}$ & $p$ Value \\
\hline Age & 1.07 & $1.01-1.13$ & 0.02 & 1.07 & $1.02-1.12$ & 0.01 \\
\hline Initial GCS score $\leq 6$ & 18.58 & $1.17-294.3$ & 0.04 & 2.85 & $0.32-25.32$ & 0.35 \\
\hline Initial abnormal pupil reaction & 0.04 & $0.001-1.04$ & 0.05 & 0.10 & $0.007-1.55$ & 0.10 \\
\hline Initial Rotterdam CT score & 4.98 & $1.40-17.78$ & 0.01 & 3.66 & $1.29-10.39$ & 0.02 \\
\hline
\end{tabular}


TABLE 5. Multivariate logistic regression analysis results for preoperative Rotterdam CT score associations with mortality and an unfavorable outcome

\begin{tabular}{|c|c|c|c|c|c|c|}
\hline \multirow[b]{2}{*}{ Variable } & \multicolumn{3}{|c|}{ Mortality } & \multicolumn{3}{|c|}{ Unfavorable Outcome } \\
\hline & OR & $95 \% \mathrm{Cl}$ & p Value & OR & $95 \% \mathrm{Cl}$ & $p$ Value \\
\hline Age & 1.05 & $1.01-1.10$ & 0.03 & 1.10 & $1.03-1.18$ & 0.007 \\
\hline Preop GCS score $\leq 6$ & 3.43 & $0.52-22.88$ & 0.20 & 3.11 & $0.43-22.54$ & 0.26 \\
\hline Preop abnormal pupil reaction & 0.57 & $0.52-6.24$ & 0.64 & 0.25 & $0.01-6.45$ & 0.41 \\
\hline Preop Rotterdam CT score & 1.77 & $0.62-5.07$ & 0.29 & 15.29 & $2.50-93.53$ & 0.003 \\
\hline
\end{tabular}

DC and that an expanded hemorrhagic contusion volume of more than $20 \mathrm{~cm}^{3}$ after DC was associated with mortality and poor 6-month GOS scores as determined by multivariable logistic regression analysis, although they did not assess for a direct association between Rotterdam CT scores and prognosis.

In the present study, we found that the initial Rotterdam CT score of a TBI patient was a significant predictor of early death after DC and that the preoperative Rotterdam CT score was a significant predictor of an unfavorable outcome at least 3 months after the time of injury. These results indicated that assessing the changes in Rotterdam CT scores over time was more effective for predicting prognosis for TBI patients who required DC than was assessing only either the initial or preoperative Rotterdam CT score. To the best of our knowledge, no previous study has assessed the sequential changes in Rotterdam CT scores with regard to the outcomes of TBI in patients who underwent DC.

We consider that the difference between initial and preoperative Rotterdam CT scores as a prognostic predictor reflects that TBI patients who experience acute deterioration after admission are more likely to survive even if they have an unfavorable outcome. None of our patients who died had a Rotterdam CT score of 6 after follow-up CT scans, and the mortality rate of TBI patients who underwent DC after a follow-up CT scan was only 9\% (2/22), which was clearly lower than the overall mortality rate of $31 \%$.

Our study had several limitations. First, this was a retrospective study of data that were previously acquired, and it has the inherent limitations of these kinds of studies. Specifically, data collected using electronic medical charts are usually less accurate and complete than data derived from prospective studies. Differences in the assessments of neurological findings could account for the observed differences in our results. Second, the number of cases was small. Currently there is no strong evidence supporting the use of DC for severe head injuries. Thus, obtaining a sufficient number of cases from only a few medical centers was difficult. Third, we did not fix the period for evaluating neurological outcomes. However, the patients included in the present study were followed up for at least 3 months after their injuries. Thus, we believe that their outcomes closely reflected the long-term outcomes after DC for TBI patients overall.

\section{Conclusions}

Our findings showed that the Rotterdam CT scores for
TBI patients who underwent DC provided important prognostic information. Initial and preoperative Rotterdam CT scores were strongly associated with, respectively, earlyperiod mortality and long-term outcomes. Thus, we can predict the prognosis for TBI patients who require DC more accurately by assessing the changes in their Rotterdam CT scores over time and combining the results with age and GCS score, taking into account that these patients may respond to postoperative supportive therapy with barbiturates and mild hypothermia $\left(32^{\circ} \mathrm{C}\right.$ to $\left.34^{\circ} \mathrm{C}\right)$, although these measures do not ensure an improved clinical outcome and their routine use is not supported by available evidence. In view of their simplicity and interobserver reproducibility, ${ }^{3}$ Rotterdam CT scores should be included as a reliable predictor in the overall clinical assessment of TBI patients prior to DC.

\section{References}

1. Aarabi B, Hesdorffer DC, Ahn ES, Aresco C, Scalea TM, Eisenberg HM: Outcome following decompressive craniectomy for malignant swelling due to severe head injury. J Neurosurg 104:469-479, 2006

2. Aarabi B, Hesdorffer DC, Simard JM, Ahn ES, Aresco C, Eisenberg HM, et al: Comparative study of decompressive craniectomy after mass lesion evacuation in severe head injury. Neurosurgery 64:927-940, 2009

3. Chun KA, Manley GT, Stiver SI, Aiken AH, Phan N, Wang $\mathrm{V}$, et al: Interobserver variability in the assessment of CT imaging features of traumatic brain injury. J Neurotrauma 27:325-330, 2010

4. Compagnone C, Murray GD, Teasdale GM, Maas AI, Esposito D, Princi $\mathrm{P}$, et al: The management of patients with intradural post-traumatic mass lesions: a multicenter survey of current approaches to surgical management in 729 patients coordinated by the European Brain Injury Consortium. Neurosurgery 57:1183-1192, 2005

5. Cooper DJ, Rosenfeld JV, Murray L, Arabi YM, Davies AR, D'Urso P, et al: Decompressive craniectomy in diffuse traumatic brain injury. N Engl J Med 364:1493-1502, 2011

6. De Bonis P, Pompucci A, Mangiola A, Paternoster G, Festa $R$, Nucci CG, et al: Decompressive craniectomy for elderly patients with traumatic brain injury: it's probably not worth the while. J Neurotrauma 28:2043-2048, 2011

7. Flint AC, Manley GT, Gean AD, Hemphill JC III, Rosenthal G: Post-operative expansion of hemorrhagic contusions after unilateral decompressive hemicraniectomy in severe traumatic brain injury. J Neurotrauma 25:503-512, 2008

8. Howard JL, Cipolle MD, Anderson M, Sabella V, Shollenberger D, Li PM, et al: Outcome after decompressive craniectomy for the treatment of severe traumatic brain injury. $\mathbf{J}$ Trauma 65:380-386, 2008

9. Huang YH, Deng YH, Lee TC, Chen WF: Rotterdam com- 
puted tomography score as a prognosticator in head-injured patients undergoing decompressive craniectomy. Neurosurgery 71:80-85, 2012

10. Huang YH, Lee TC, Lee TH, Liao CC, Sheehan J, Kwan AL: Thirty-day mortality in traumatically brain-injured patients undergoing decompressive craniectomy. J Neurosurg 118:1329-1335, 2013

11. Hukkelhoven CW, Steyerberg EW, Habbema JD, Farace E, Marmarou A, Murray GD, et al: Predicting outcome after traumatic brain injury: development and validation of a prognostic score based on admission characteristics. J Neurotrauma 22:1025-1039, 2005

12. Jaeger M, Soehle M, Meixensberger J: Effects of decompressive craniectomy on brain tissue oxygen in patients with intracranial hypertension. J Neurol Neurosurg Psychiatry 74:513-515, 2003

13. Katsnelson M, Mackenzie L, Frangos S, Oddo M, Levine JM, Pukenas B, et al: Are initial radiographic and clinical scales associated with subsequent intracranial pressure and brain oxygen levels after severe traumatic brain injury? Neurosurgery 70:1095-1105, 2012

14. Lazaridis C, Czosnyka M: Cerebral blood flow, brain tissue oxygen, and metabolic effects of decompressive craniectomy. Neurocrit Care 16:478-484, 2012

15. Leitgeb J, Mauritz W, Brazinova A, Janciak I, Majdan M, Wilbacher I, et al: Outcome after severe brain trauma due to acute subdural hematoma. J Neurosurg 117:324-333, 2012

16. Lingsma HF, Roozenbeek B, Steyerberg EW, Murray GD, Maas AI: Early prognosis in traumatic brain injury: from prophecies to predictions. Lancet Neurol 9:543-554, 2010

17. Lobato RD, Gomez PA, Alday R, Rivas JJ, Dominguez J, Cabrera A, et al: Sequential computerized tomography changes and related final outcome in severe head injury patients. Acta Neurochir (Wien) 139:385-391, 1997

18. Maas AI, Hukkelhoven CW, Marshall LF, Steyerberg EW: Prediction of outcome in traumatic brain injury with computed tomographic characteristics: a comparison between the computed tomographic classification and combinations of computed tomographic predictors. Neurosurgery 57:11731182,2005

19. Maas AI, Steyerberg EW, Butcher I, Dammers R, Lu J, Marmarou A, et al: Prognostic value of computerized tomography scan characteristics in traumatic brain injury: results from the IMPACT study. J Neurotrauma 24:303-314, 2007

20. Marmarou A, Lu J, Butcher I, McHugh GS, Murray GD, Steyerberg EW, et al: Prognostic value of the Glasgow Coma Scale and pupil reactivity in traumatic brain injury assessed pre-hospital and on enrollment: an IMPACT analysis. J Neurotrauma 24:270-280, 2007

21. Marshall LF, Marshall SB, Klauber MR, Clark MB: A new classification of head injury based on computerized tomography. J Neurosurg 75 Suppl:S14-S22, 1991

22. Mata-Mbemba D, Mugikura S, Nakagawa A, Murata T, Ishii
K, Li L, et al: Early CT findings to predict early death in patients with traumatic brain injury: Marshall and Rotterdam CT scoring systems compared in the major academic tertiary care hospital in northeastern Japan. Acad Radiol 21:605611,2014

23. Nelson DW, Nyström H, MacCallum RM, Thornquist B, Lilja A, Bellander BM, et al: Extended analysis of early computed tomography scans of traumatic brain injured patients and relations to outcome. J Neurotrauma 27:51-64, 2010

24. Olivecrona M, Rodling-Wahlström M, Naredi S, Koskinen LO: Effective ICP reduction by decompressive craniectomy in patients with severe traumatic brain injury treated by an ICP-targeted therapy. J Neurotrauma 24:927-935, 2007

25. Reithmeier T, Löhr M, Pakos P, Ketter G, Ernestus RI: Relevance of ICP and ptiO2 for indication and timing of decompressive craniectomy in patients with malignant brain edema. Acta Neurochir (Wien) 147:947-952, 2005

26. Servadei F, Nasi MT, Giuliani G, Cremonini AM, Cenni P, Zappi D, et al: CT prognostic factors in acute subdural haematomas: the value of the 'worst' CT scan. Br J Neurosurg 14:110-116, 2000

27. Shahin H, Gopinath SP, Robertson CS: Influence of alcohol on early Glasgow Coma Scale in head-injured patients. J Trauma 69:1176-1181, 2010

28. Stiefel MF, Heuer GG, Smith MJ, Bloom S, Maloney-Wilensky E, Gracias VH, et al: Cerebral oxygenation following decompressive hemicraniectomy for the treatment of refractory intracranial hypertension. J Neurosurg 101:241-247, 2004

29. Stocchetti N, Pagan F, Calappi E, Canavesi K, Beretta L, Citerio G, et al: Inaccurate early assessment of neurological severity in head injury. J Neurotrauma 21:1131-1140, 2004

\section{Disclosure}

The authors report no conflict of interest concerning the materials or methods used in this study or the findings specified in this paper.

\section{Author Contributions}

Conception and design: Fujimoto. Acquisition of data: Fujimoto. Analysis and interpretation of data: Fujimoto. Drafting the article: Fujimoto. Critically revising the article: Miura, Otsuka, Kuratsu. Reviewed submitted version of manuscript: Miura, Otsuka, Kuratsu. Approved the final version of the manuscript on behalf of all authors: Fujimoto. Statistical analysis: Fujimoto.

\section{Correspondence}

Kenji Fujimoto, Department of Neurosurgery, Faculty of Life Sciences, Kumamoto University School of Medicine, 1-1-1, Honjo, Chuo-ku, Kumamoto 861-8556, Japan. email: schwarz k1216@yahoo.co.jp. 